Laser Chem., 1999, Vol. 19, pp. 45-49

Reprints available directly from the publisher Photocopying permitted by license only
(C) 1999 OPA (Overseas Publishers Association) N.V.

Published by license under

the Harwood Academic Publishers imprint,

part of The Gordon and Breach Publishing Group.

Printed in India.

\title{
VIBRATIONAL COOLING AFTER PHOTO ISOMERISATION: FIRST APPLICATION OF A NOVEL INTRAMOLECULAR THERMOMETER
}

\author{
P. HAMM*, S. OHLINE ${ }^{\dagger}$, M. ZUREK and T. RÖSCHINGER \\ Institut für Medizinische Optik, Ludwig Mximilians Universität München, \\ Barbarastr. 16, 80797 München, Germany
}

(Received 7 April 1997)

Femtosecond VIS-pump-IR-probe experiments (time resolution: $300 \mathrm{fs}$, detectable absorption change: $5 \cdot 10^{-5}$, frequency range: $1000-1800 \mathrm{~cm}^{-1}$ ) were applied to the ultrafast photo isomerisation of azobenzene and the protonated Schiff base of retinal. The energy of the pump photon heats the vibrational system of the molecule, leading to pronounced anharmonic frequency shifts which are dominated by the anharmonic coupling between the investigated high frequency modes to hot low frequency modes. A model taking into account this intramolecular anharmonicity and assuming a well defined intramolecular temperature gives a good fit of the experimental data.

Keywords: Femtosecond infrared spectroscopy; cis-trans isomerisation; vibrational cooling; azobenzene; retinal

\section{INTRODUCTION}

Mainly due to its importance in many biological systems, photon induced cis-trans-isomerisation reactions have been widely studied. Briefly, the reaction can be described as follows: After excitation, the molecules reach a region on the excited state potential surface in a

\footnotetext{
* Corresponding author. Department of Chemistry, University of Pennsylvania, Philadelphia, PA 19104, USA.

${ }^{\dagger}$ Present address: Department of Chemistry, Wellesley College, Wellesley, MA02181, USA.
} 
twisted configuration from where the transition to the $S_{0}$ surface can occur, transferring the excess energy to coupled vibrational modes. The initially, non-thermal energy distribution is equilibrated by the IVR process, leading to remarkable intramolecular temperatures $(\leq 1000 \mathrm{~K})$. In order to get new insights into the vibrational energy after the internal conversion process we have investigated two model systems by femtosecond IR experiments: azobenzene and retinal in solution $[1,2]$.

\section{MODEL}

Due to the equidistant energy levels of a harmonic oscillator and vibrational excited state absorption, no IR absorption changes are expected upon vibrational excitation as long as anharmonicity is not explicitly taken into account. When considering anharmonicity, the transition frequency $\omega_{k}$ of a mode depends on the excitation level of the mode itself (diagonal anharmonicity) and also on the excitation levels of the remaining 'bath' mode (off-diagonal anharmonicity) [3]:

$$
\omega_{k}=\omega_{k, 0}+2 x_{k k} n_{k}+\sum_{i \neq k} x_{i k} n_{i}
$$

$\omega_{k, 0}$ is the transition frequency in the ground state, $n_{k, i}$ the excitation of mode $k, i$ and $x_{k k}$ and $x_{i k}$ are the diagonal and the off-diagonal anharmonic constants, respectively. Since in general, most of the $x_{k k}$ and $x_{i k}$ are negative, Eq. (1) results in a red shift of mode $k$. As shown in [1] for molecules of the size of azobenzene, having mostly delocalized modes, the off-diagonal anharmonicity is dominating the anharmonic effects. However, since the anharmonic constants are not known in detail, a simplifying model is developed: It is assumed that the off-diagonal anharmonic constant $x_{i k}$ between mode $k$ and the bath mode $i$ is proportional to the frequency $\omega_{i}: x_{i k}=x \cdot \omega_{i}$, in rough agreement with the anharmonic constants which have been calculated for benzene [4]. From this formula, one can conclude that the mean total frequency shift $\Delta \omega_{k}$ of mode $k$ is proportional to the total intramolecular vibrational energy: $\Delta \omega_{k}=x \cdot E_{\mathrm{vib}}$, regardless of the detailed energy distribution. Thus, time resolved IR spectroscopy of 
large molecules is mostly insensitive to IVR while it provides a measure of the total vibrational energy. This also justifies the approach to describe the intramolecular energy distribution by a Boltzmann distribution, i.e., by an intramolecular temperature, even when is not clear whether the IVR-process is completed. A good qualitative agreement between the experimental data and the model is obtained under these model assumptions, as shown in Figure 1 for azobenzene.

\section{APPLICATION TO RETINAL}

As an application, the protonated Schiff base of retinal (PSBR) in ethanol was investigated in the spectral range of the $\mathrm{C}-\mathrm{C}$, the $\mathrm{C}=\mathrm{C}$ and $\mathrm{C}=\mathrm{N}$ modes [2] (see Fig. 2). With the electronic decay these

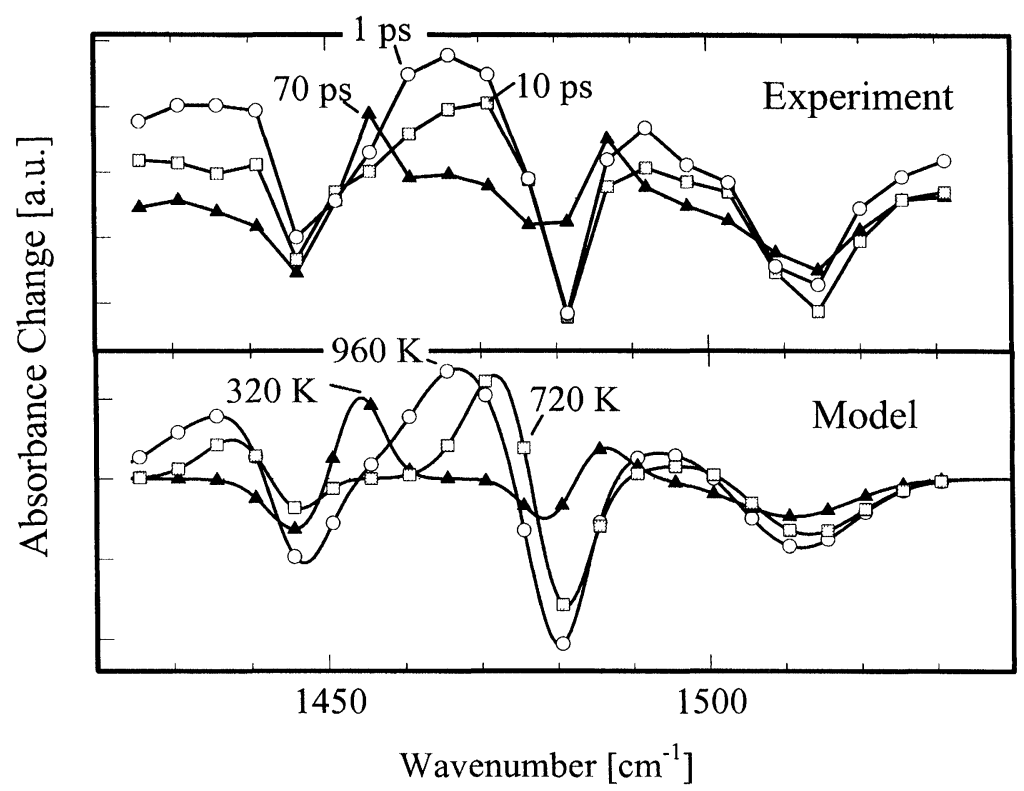

FIGURE 1 Transient IR absorption changes of cis-azobenzene upon switching the molecules from the cis to the trans configuration with a short light pulse at $407 \mathrm{~nm}$ (top). The model spectra (bottom) taking into account the intramolecular anharmonicity and assuming a well defined intramolecular temperature qualitatively fit the experimental data very well. 


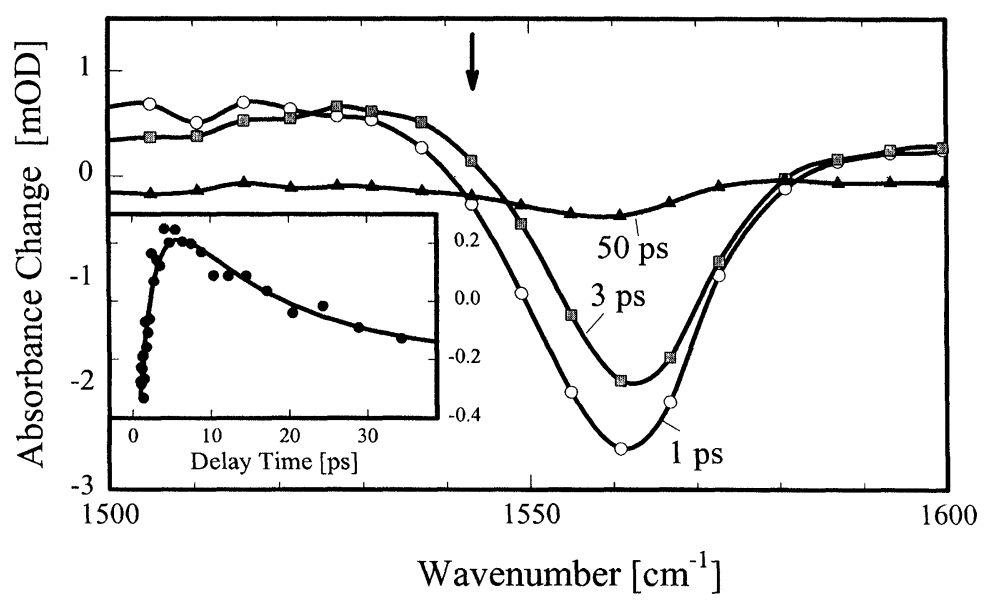

FIGURE 2 Transient difference spectra of PSBR. The difference between the $1 \mathrm{ps}$ and the $3 \mathrm{ps}$ spectrum mainly reflects the recover of the bleached $\mathrm{C}=\mathrm{C}$ stretching mode due to the electronic $S_{1}-S_{0}$ transition, which occurs on a time scale of $2 \mathrm{ps}$. A detailed analysis shows that initially the band shows up ca. $15 \mathrm{~cm}^{-1}$ lower than the frequency found for the cold molecules [2]. Insert: Temporal evolution at a frequency position marked by the arrow: The initial fast absorption rise reflects the formation of the hot band while the subsequent slow decrease is caused by the cooling of the molecules due to the intermolecular energy transfer to the medium.

vibrational modes are initially formed at a frequency $15 \mathrm{~cm}^{-1}$ lower than found in the cold molecules. In a steady-state absorption spectrum of PSBR, a frequency shift of $1.8 \mathrm{~cm}^{-1}$ is obtained when heating the sample from 300 to $340 \mathrm{~K}$. This value can be used to estimate the anharmonic constant $x$ which subsequently is applied to extrapolate the anharmonic effect at higher temperatures. This 'calibration' of the thermometer yields an initial temperature of $550 \mathrm{~K}$ for the observed $15 \mathrm{~cm}^{-1}$. Interestingly, the anharmonic effect in the bacteriorhodopsin, i.e., the chromophore in its native protein surrounding, is considerably smaller: For the cis- $\mathrm{C}=\mathrm{N}$ mode a frequency shift of only $5 \mathrm{~cm}^{-1}$ between $1.5 \mathrm{ps}$ spectrum and the $9 \mathrm{ps}$ spectrum was found [5], pointing to a temperature of $400 \mathrm{~K}$. Part of the difference can be explained by the lower excitation energy used in these experiments and the fact that ca. $0.5 \mathrm{eV}$ is stored in the cis-photo product of BR (in contrast to our experiments). 


\section{References}

[1] Hamm, P., Ohline, S. and Zinth, W. (1997). J. Chem. Phys., 106, 519.

[2] Hamm, P., Zurek, M. Röschinger, T., Patzelt, H., Oesterhelt, D. and Zinth, W. (1997). Chem. Phys. Lett., 268, 180.

[3] Herzberg, G.. Molecular Spectra and Molecular Structure. II. Infrared and Raman Spectra of Polyatomic Molecules (Van Nostrand Reinhold Company, New York, 1945).

[4] Maslen, P. E., Handy, N. C., Amos, R. D and Jayatilaka, D. (1992). J. Chem. Phys., 97, 4233.

[5] Diller, R., Maiti, S., Walker, G. C., Cowen, B. R., Pippenger, R., Bogomolni, R. A. and Hochstrasser, R. M. (1995). Chem. Phys. Lett., 241, 109. 